\title{
Dermatitis atópica en niños: estudio comparativo en dos grupos etarios
}

\author{
PABLO G. DEI-CAS ${ }^{1}$, MARÍA KARINA ACUÑA ${ }^{2}$, IGNACIO DEI-CAS ${ }^{2}$ \\ 1. Médico especialista en Pediatría. \\ 2. Médico especialista en Dermatología. \\ Unidad Sanitaria 8 de Octubre. Quilmes, Buenos Aires. Argentina.
}

\begin{abstract}
Atopic dermatitis in children: a comparative survey among 2 age groups

Introduction: Prevalence of atopic dermatitis (AD) has increased steadily. Risk factors are being researched worldwide. There is little data available in Argentina. Objetives y Methods: This study included 722 children $\geq 12$ and $<60$ months of age residing in an urban community in Buenos Aires. UK's Working Party's criteria were used to establish the prevalence of AD. The objectives were a) to establish the prevalence of AD in children $<36$ and $\geq 36$ months of age, b) evaluate gender differences in prevalence, and c) evaluate association between $\mathrm{AD}$ and the following variables: family history of atopia and degree of consanguinity; number of people living together $\leq 4$; use of synthetic clothing in direct contact with the skin; tobacco use within the home; use of rugs/carpeting within the home; environmental contaminating factors in each age group. $\chi^{2}$ was used to calcúlate associations in a logistic regression model. Results: Prevalence of AD was 31,3\% (CI: 27,235,6 ) among children $<36$ months and 49,6\% (CI: 43,1-56,1) among children $\geq 36$ months. AD was more prevalent among younger males. Family history of atopia, use of synthetic material, and living near a factory were associated to $\mathrm{AD}$ in both age groups. Using rugs/carpeting inside the house was associated to the disease only in younger children. Conclusion: An elevated prevalence of $\mathrm{AD}$ was found in this low-income population. It predominates among older children, which differs from commonly accepted findings. Not all evaluated risk factors were associated to the disease.

(Key words: Atopic dermatitis, prevalence, risk factors, atopia).

Rev Chil Pediatr 2011; 82 (5): 410-418
\end{abstract}

\section{RESUMEN}

Introducción: La prevalencia de la dermatitis atópica (DA) ha aumentado sostenidamente y sus factores de riesgo son investigados mundialmente. En la Argentina, sin embargo, existen escasos datos disponibles. Objetivos y Métodos: Estudio de prevalencia, de corte transversal que incluyó 722 niños $\geq 12$ y $<60$ meses de edad pertenecientes a una comunidad urbana bonaerense. Se utilizaron los criterios del the U.K. Working

Trabajo recibido el 07 de enero de 2011, devuelto para corregir el 19 de marzo de 2011, segunda versión el 20 de julio de 2011, aceptado para publicación el 22 de agosto de 2011.

Correspondencia a:

Pablo Dei-Cas.

e-mail: pgdeicas@yahoo.com.ar 
Party's para definir DA. Los objetivos fueron establecer la prevalencia de la DA en niños $<36$ meses y $\geq 36$ meses de edad, conocer si existen diferencias de prevalencia según sexo, y evaluar asociaciones entre DA y las siguientes variables: presencia de antecedentes familiares de atopía según grado, número de convivientes $\leq 4$, uso de ropa sintética en contacto directo con la piel, consumo de tabaco en el interior de la vivienda, alfombra/s dentro del hogar y factores contaminantes medioambientales en cada grupo etario. Se utilizó la prueba de $\chi^{2}$ para calcular asociaciones, que de ser significativas se incluyeron en un modelo de regresión logística. Resultados: La prevalencia de DA fue del 31,3\% (IC: 27,2-35,6) en los < 36 meses y 49,6\% (IC: 43,1-56,1) en los $\geq 36$ meses. La DA prevaleció en el sexo masculino en los niños más pequeños. El AF de atopía, el uso de ropa de material sintético y habitar cerca de una fábrica se relacionaron con la DA en ambos grupos etarios, en tanto poseer alfombra/s dentro del hogar se asoció con la enfermedad sólo en los niños pequeños. Conclusión: Hallamos una elevada prevalencia de DA en esta población de bajos recursos, y que la DA predominó en niños mayores, resultados que se contraponen a lo actualmente aceptado, y que no todos los factores de riesgo investigados se asociaron con la enfermedad.

(Palabras clave: Dermatitis atópica, prevalencia, factores de riesgo, atopía).

Rev Chil Pediatr 2011; 82 (5): 410-418

\section{Introducción}

La dermatitis atópica (DA) es una enfermedad cutánea inflamatoria crónica frecuente en la edad pediátrica. Su evolución suele ser autolimitada y se caracteriza por alternar períodos con remisiones y recaídas. Su etiología es desconocida, encontrándose implicados en su desarrollo tanto factores genéticos como ambientales.

Estudios epidemiológicos a nivel mundial demuestran un aumento sostenido de la prevalencia de DA en las últimas décadas, habiéndose incrementado la misma en algunos países más de dos veces desde 1960 hasta la actua$\operatorname{lidad}^{1,2}$. Se conoce que la prevalencia de DA disminuye al aumentar la edad del paciente y tiende a desaparecer al iniciarse la escolaridad.

De acuerdo a nuestro conocimiento, hasta el año 2004, en la Argentina no se disponía de datos epidemiológicos reportados en la bibliografía nacional acerca de esta enfermedad ${ }^{3}$.

\section{Objetivos}

1) Estimar la prevalencia de DA en niños de bajos recursos correspondientes a 2 grupos etarios ( $<3$ y $\geq 3$ años de edad) que habitan en el sur del conurbano bonaerense; 2) Conocer si existen diferencias de prevalencia de DA según sexo en cada grupo; 3) Analizar si existen diferencias entre los niños pequeños y los de mayor edad en relación a los siguientes facto- res de riesgo: antecedentes familiares $(\mathrm{AF}) \mathrm{de}$ atopía en familiares de primero $\left(\mathrm{AF} 1^{\circ}\right)$, segundo grado $\left(\mathrm{AF} 2^{\circ}\right)$ y primero y segundo grado $\left(\mathrm{AF} 1^{\circ}\right.$ y $\left.2^{\circ}\right)$, número de convivientes, uso de ropa sintética en contacto directo con la piel, consumo de tabaco en el interior de la vivienda, presencia de alfombra/s dentro del hogar y factores contaminantes medioambientales.

\section{Pacientes y Método}

Estudio descriptivo de corte transversal realizado en una muestra no probabilística consecutiva constituída por niños de ambos sexos con edades $\geq 12$ y $<60$ meses, sin antecedentes patológicos conocidos, período abril 2008-mayo 2009.

Los pacientes concurrieron para su control de salud a una Unidad Sanitaria, cuya área programática abarca una superficie de $0,96 \mathrm{~km}^{2}, \mathrm{y}$ se encuentra ubicada en San Francisco Solano, localidad del partido de Quilmes (sur del conurbano bonaerense).

El área programática de este Centro de Atención Primaria cuenta, de acuerdo a cifras del último Censo Nacional, con una población de 893 niños con edades comprendidas entre los 12 y 60 meses.

La participación de los pacientes en el estudio fue voluntaria, habiéndose alcanzado un 93\% de aceptación. Previo a la inclusión del 
paciente en el presente estudio, se hizo llegar al familiar responsable el consentimiento informado, quien de aceptar participar lo firmaba en conformidad. La muestra en cuestión logró captar al 81\% del total de niños del grupo etario elegido que habita el área programática del Centro de Salud.

\section{Criterios de exclusión}

Pacientes con enfermedades de base, y aquéllos cuyo familiar responsable no autorizaba su participación en la investigación.

Pacientes cuyo acompañante no se encontraba en condiciones de brindar información confiable.

\section{Criterio diagnóstico}

Se definió DA de acuerdo al UK Working Party's Diagnostic Criteria for Atopic Dermatitis, que incluye el hallazgo de prurito sumado a la presencia de por lo menos 3 de los siguientes criterios: xerodermia en el último año, eczema en frente, mejillas y parte externa de miembros al momento de la consulta, antecedente familiar de atopía, e historia personal de eczema durante el último año ${ }^{4}$.

El diagnóstico de esta patología fue llevado a cabo por médicos dermatólogo y pediatra conjuntamente.

\section{Factores de riesgo de DA evaluados:}

Los datos fueron obtenidos al momento de la consulta a través del interrogatorio efectuado al adulto acompañante.

- Antecedentes familiares de atopía: presencia de DA, rinitis, asma, urticaria alimentaria o conjuntivitis alérgica en familiares directos. Se consideraron familiares de $1^{\circ}$ grado a madre, padre y hermanos, y familiares de $2^{\circ}$ grado a tíos y abuelos.

- Núcleo familiar pequeño: $\leq 4$ personas en el ámbito del hogar.

- Uso de ropa de material sintético: fibras sintéticas presentes en contacto directo con la piel al momento de la consulta.

- Presencia de alfombras dentro del hogar: 1 o más alfombras tipo carpeta o piso alfombrado en 1 o más ambientes.
- Consumo de tabaco en el interior de la vivienda: consumo de cigarrillos/pipa por familiares directos dentro de la casa.

- Factores contaminantes medioambientales:

- Vivienda cercana a una avenida: situada a menos de 300 metros.

- Vivienda cercana a un arroyo contaminado: situada a menos de 300 metros.

- Vivienda próxima a una industria (fábrica): situada a menos de 300 metros.

\section{Análisis estadístico}

El tamaño muestral se calculó mediante la utilización del programa estadístico Stat Calc.

Los datos recogidos fueron cargados y procesados con el programa estadístico Epi Info $^{\mathrm{TM}}$, versión 3.3.2. La no existencia de los factores de riesgo investigados constituyó la hipótesis nula que se intentó rechazar con un error alfa del $5 \%$. Se calcularon medias y DS cuando correspondiera. Se utilizó la prueba estadística de $\chi^{2}$ para comparar diferencias entre los grupos con y sin DA. Las variables que resultaron estadísticamente significativas fueron incluidas en un modelo de análisis multivariado. Los resultados se expresan en Odds ratio (OR) y sus respectivos intervalos de confianza (IC) del 95\%. Se consideró estadísticamente significativo si $\mathrm{p}<0,05$.

Se indicó tratamiento a los pacientes con diagnóstico de DA.

\section{Resultados}

Se evaluaron 781 niños, de los cuales 722 (333 de sexo femenino y 389 de sexo masculino) conformaron la muestra. La edad media de los sujetos evaluados fue de 29,2 $\pm 14,6$ meses.

La DA se diagnosticó en el 37,4\% (IC95\%: 33,9 - 41,1) de los pacientes evaluados, estando presente en el 31,3\% (IC95\%: 27,2 - 35,6) de los menores de 3 años y en el 49,6\% (IC95\%: 43,1 - 56,1) de los mayores. Encontramos que la DA prevaleció en el sexo masculino en el grupo de niños más pequeños (p: 0,02) sin haberse hallado diferencias en los mayores de 3 años. Las características de la muestra por grupos etarios se detallan en la tabla 1. Las asociaciones entre DA y los factores de riesgo 
Tabla 1. Características de la muestra

\begin{tabular}{|c|c|c|c|c|}
\hline \multirow[t]{3}{*}{ Característica } & \multicolumn{4}{|c|}{ Edad } \\
\hline & \multicolumn{2}{|c|}{$\begin{array}{c}<36 \text { meses } \\
(\mathrm{n}: 480)\end{array}$} & \multicolumn{2}{|c|}{$\begin{array}{c}\geq 36 \text { meses } \\
\quad(n: 242)\end{array}$} \\
\hline & $\mathbf{n}$ & $\%$ & $\mathbf{n}$ & $\%$ \\
\hline $\begin{array}{l}\text { Sexo } \\
\text { - fem } \\
\text { - masc }\end{array}$ & $\begin{array}{l}211 \\
269\end{array}$ & $\begin{array}{l}44 \\
56\end{array}$ & $\begin{array}{l}122 \\
120\end{array}$ & $\begin{array}{l}50,4 \\
49,6\end{array}$ \\
\hline $\begin{array}{l}\text { AF atopía } \\
\text { - } A F 1^{\circ} \\
\text { - } A F 2^{\circ} \\
\text { - } A F 1^{\circ} \text { Y } 2^{\circ}\end{array}$ & $\begin{array}{r}125 \\
46 \\
15\end{array}$ & $\begin{array}{r}30,4 \\
11,2 \\
3,6\end{array}$ & $\begin{array}{r}52 \\
32 \\
9\end{array}$ & $\begin{array}{r}28,1 \\
17,3 \\
4,9\end{array}$ \\
\hline Convivientes $\leq 4$ & 200 & 41,7 & 104 & 43 \\
\hline Ropa sintética & 124 & 25,8 & 80 & 33,1 \\
\hline Consumo tabaco en la vivienda & 285 & 59,4 & 153 & 63,2 \\
\hline Alfombras & 113 & 23,5 & 64 & 26,4 \\
\hline $\begin{array}{l}\text { Contaminantes medioambientales } \\
\text { - Avda }<300 \mathrm{~m} \\
\text { - Arroyo }<300 \mathrm{~m} \\
\text { - Fábrica }<300 \mathrm{~m}\end{array}$ & $\begin{array}{l}276 \\
163 \\
137\end{array}$ & $\begin{array}{l}57,5 \\
34 \\
28,6\end{array}$ & $\begin{array}{r}158 \\
89 \\
67\end{array}$ & $\begin{array}{l}65,3 \\
36,8 \\
27,7\end{array}$ \\
\hline
\end{tabular}

Tabla 2. Asociaciones entre DA y variables evaluadas en niños $<36$ meses

\begin{tabular}{|llll|}
\hline & OR & IC 95\% & \multicolumn{1}{c|}{ p } \\
\hline AF1 $^{\circ}$ & 3,2 & $2-5,1$ & 0,0000 \\
\hline AF2 $^{\circ}$ & 2,7 & $1,5-5,1$ & 0,0014 \\
\hline AF $1{ }^{\circ} \mathrm{y} 2^{\circ}$ & 3,8 & $1,3-10,9$ & 0,012 \\
\hline Convivientes $\leq 4$ & 1,1 & $0,7-1,6$ & NS \\
\hline Ropa sintética & 3,2 & $2,1-4,9$ & 0,0000 \\
\hline Consumo de tabaco en la vivienda & 1,5 & $1-2,2$ & 0,03 \\
\hline Alfombra & 2 & $1,3-3,1$ & 0,001 \\
\hline Avenida $<300 \mathrm{~m}$ & 1,1 & $0,7-1,6$ & $\mathrm{NS}$ \\
\hline Arroyo contaminado $<300 \mathrm{~m}$ & 1,3 & $0,9-2$ & $\mathrm{NS}$ \\
\hline Fábrica $<300 \mathrm{~m}$ & 2,1 & $1,4-3,1$ & 0,0004 \\
\hline
\end{tabular}

Tabla 4. Análisis de regresión logística en niños $<36$ meses

\begin{tabular}{|lrrl|}
\hline & OR & IC 95\% & p \\
\hline AF1 $^{\circ}$ & 5,8 & $3,3-10,1$ & 0,0000 \\
\hline AF2 $^{\circ}$ & 6,5 & $3,1-13,8$ & 0,0000 \\
\hline AF $1{ }^{\circ} \mathrm{y} 2^{\circ}$ & 13,4 & $4-44,1$ & 0,0000 \\
\hline Ropa sintética & 2,6 & $1,5-4,5$ & 0,0008 \\
\hline Consumo de tabaco en la vivienda & 1,6 & $0,9-2,6$ & NS \\
\hline Alfombra & 1,9 & $1,1-3,3$ & 0,027 \\
\hline Fábrica $<300 \mathrm{~m}$ & 2,2 & $1,3-3,6$ & 0,0034 \\
\hline
\end{tabular}

evaluados según grupo etario se detallan en las tablas 2 y 3 .

El análisis multivariado confirmó que los factores de riesgo que se relacionaron con el diagnóstico de DA en los niños pequeños fueron la presencia de AF de atopía en familiares de $1^{\circ}$ grado, de $2^{\circ}$ grado y conjuntamente de $1^{\circ}$ y $2^{\circ}$ grado, el uso de ropa sintética en contacto directo con la piel, poseer alfombra/s dentro del hogar y vivir en las cercanías de una fábrica (tabla 4). En los niños mayores sólo el antecedente familiar de atopía en familiares de $1^{\circ}$ grado y en $1^{\circ}$ y $2^{\circ}$ grado conjuntamente, el uso de ropa sintética y habitar a menos de 300 metros de una fábrica se relacionaron estadísticamente con la DA (tabla 5).

Tabla 3. Asociaciones entre DA y variables evaluadas en niños $\geq 36$ meses

\begin{tabular}{|llll|}
\hline & OR & IC 95\% & \multicolumn{1}{c|}{ p } \\
\hline AF1 ${ }^{\circ}$ & 5,5 & $2,6-11,5$ & 0,00000 \\
\hline AF2 ${ }^{\circ}$ & 1,7 & $0,8-3,6$ & NS \\
\hline AF $1{ }^{\circ}$ y2 ${ }^{\circ}$ & 9,2 & $1,1-74,9$ & 0,014 \\
\hline Convivientes $\leq 4$ & 0,7 & $0,4-1,2$ & NS \\
\hline Ropa sintética & 1,9 & $1,1-3,2$ & 0,01 \\
\hline $\begin{array}{l}\text { Consumo de tabaco } \\
\text { en la vivienda }\end{array}$ & 1,3 & $0,8-2,2$ & NS \\
\hline Alfombra & 1,6 & $0,9-2,8$ & NS \\
\hline Avenida $<300$ m & 0,7 & $0,4-1,1$ & NS \\
\hline $\begin{array}{l}\text { Arroyo contaminado } \\
<300 ~ m\end{array}$ & 1,4 & $0,8-2,4$ & NS \\
\hline \begin{tabular}{l} 
Fábrica $<300$ m \\
\hline
\end{tabular} & 3 & $1,6-5,4$ & 0,0001 \\
\hline
\end{tabular}

Tabla 5. Análisis de regresión logística en niños $\geq \mathbf{3 6}$ meses

\begin{tabular}{|lrrr|}
\hline & OR & IC 95\% & p \\
\hline AF1 ${ }^{\circ}$ & 11,8 & $5-27,9$ & 0,0000 \\
\hline AF $1^{\circ} \mathrm{y} 2^{\circ}$ & 30,2 & $3-269,8$ & 0,0023 \\
\hline Ropa sintética & 3,6 & $1,6-8,1$ & 0,0022 \\
\hline Fábrica $<300 \mathrm{~m}$ & 2,6 & $1,2-5,8$ & 0,017 \\
\hline
\end{tabular}




\section{Discusión}

El estudio epidemiológico de una enfermedad requiere de una definición de la misma y de la posibilidad de contar con elementos diagnósticos confiables y validados. La DA es una entidad con múltiples manifestaciones clínicas que varían a través del tiempo, es por ello que la dificultad para certificar su diagnóstico ha motivado el desarrollo de diferentes metodologías basadas en criterios clínicos y de laboratorio.

La prevalencia de la DA ha sido estudiada en diferentes poblaciones a nivel mundial, presentando una amplia variabilidad, con cifras reportadas que oscilan entre el $0,73 \%$ en un estudio realizado en un área rural de Tanzania ${ }^{1}$ y el $41,1 \%$ en una investigación llevada a cabo por nuestro grupo de trabajo ${ }^{5}$.

En el presente estudio, la prevalencia de DA fue del $31,3 \%$ en niños $<3$ años y del $49,6 \%$ en los niños mayores, cifras llamativamente elevadas en relación a la literatura, e incluso superiores a los datos argentinos obtenidos de las ciudades de Buenos Aires y Rosario y publicados en el estudio internacional de Williams y col, cuyo promedio para nuestro país resultó del 7,5\% $\%^{6}$. Dicho estudio multicéntrico evaluó mediante la utilización de un cuestionario 256410 niños de 2 grupos etarios (6 a 7 y 13 a 14 años), provenientes de 56 países $^{6}$.

Para explicar el amplio rango de prevalencia de DA entre los distintos estudios podrían plantearse diferentes hipótesis.

La edad de los pacientes seleccionados es una variable metodológica capaz de arrojar diferencias de prevalencia de la enfermedad ya que la DA presenta una tendencia natural a remitir con la edad. El concepto clásicamente aceptado es que la DA desaparece en un alto porcentaje de los casos con el correr de los años ${ }^{1,2}$, sin embargo, no existe consenso que determine a partir de qué edad se hace evidente este cambio. Williams y Wüthrich encontraron que la enfermedad remite a los 10 años de edad entre el 50 y $70 \%$ de los pacientes ${ }^{1}$ Illi y col hallaron en un estudio prospectivo longitudinal que la prevalencia de DA disminuye y se estabiliza a partir de los 3 años de edad ${ }^{7}$. Sorpresiva y contrariamente a lo esperado, en nuestra muestra la prevalencia de DA fue superior en el grupo de niños mayores de 3 años, no pudiendo encontrar explicación a tal hallazgo. Estos resultados obligarían a llevar a cabo nuevos estudios, con mayor cantidad de casos y en poblaciones de similares características.

La ausencia de un criterio diagnóstico único y universalmente aceptado hace que los autores escojan arbitrariamente los criterios diagnósticos más confiables a su juicio o con los que se encuentran más familiarizados. En el año 2005 Johnke y col${ }^{8}$, publican en el British Journal of Dermatology un artículo que evaluó prospectivamente la prevalencia de DA en niños menores de 18 meses mediante la utilización de los criterios de Hanifin y Rajka, de Schultz Larsen, del UK Working Party, del Danish Allergy Research Centre y del Visible eczema no encontrando diferencias de prevalencia estadísticamente significativas, independientemente del método diagnóstico utilizado. Este hallazgo nos permitiría inferir que la alta prevalencia de DA que encontramos en nuestra investigación no sería consecuencia de los criterios diagnósticos escogidos.

Otro ítem metodológico destacable, capaz de arrojar cifras de prevalencia disímiles, sería la gran variabilidad en el diseño de los estudios publicados, ya que se dispone de trabajos tanto prospectivos como retrospectivos que utilizan cuestionarios ${ }^{6,9,10}$ o el examen clínico ${ }^{7,11-13}$ para arribar al diagnóstico de esta dermatosis.

Williams ${ }^{14}$, señala también, que la prevalencia de DA puede variar considerablemente de un país a otro, e incluso dentro de un mismo país, concepto que explicaría los diferentes resultados entre nuestro trabajo y los datos argentinos publicados en el estudio internacional ${ }^{6}$.

La prevalencia de DA también estaría determinada por la presencia de AF de atopía en la muestra evaluada, como publican recientemente Brydensholt y cols, en un estudio llevado a cabo en Dinamarca en el que encontraron cifras de DA a los 3 años de edad del $44 \%$ en hijos de madres asmáticas ${ }^{13}$. El AF de atopía se presentó en el 74,9\% de los niños con DA de nuestra muestra, pudiendo explicar así la alta prevalencia de DA que encontramos.

La contribución de la etnia en la génesis de 
la DA es controvertida. Williams y cols, encontraron que el riesgo de desarrollar DA en niños de raza negra, nacidos en Gran Bretaña es mayor que para los caucásicos ${ }^{15}$. Coincidentemente, otros autores encontraron mayores prevalencias de DA en negros y asiáticos ${ }^{10,16}$. Neame y cols, en contraposición, no encontraron que la misma se relacione con la raza ${ }^{17}$.

Se postula que la migración sería otro factor de riesgo para el desarrollo de DA, pudiendo asociarse con la exposición a nuevos alergenos ${ }^{14}$. Si bien no constituyó un objetivo de nuestro estudio evaluar el grupo étnico de los pacientes, podríamos especular que la alta prevalencia de DA que encontramos sería consecuencia de que una parte importante de las familias del área geográfica evaluada constituyen una población golondrina, proveniente en su mayoría, de países limítrofes.

La existencia de asociación entre la DA y el sexo es discutida. Existen estudios reportados que no encontraron diferencias de prevalencia según sexo ${ }^{13,18,19}$. Moore $^{10}$ y Harris $^{20}$, entre otros, constataron predominio de DA en varones. En la bibliografía también se reportan cifras que muestran mayoría de niñas con la patología en estudio ${ }^{6,9}$.

Nuestros resultados mostraron predominio de DA en el sexo masculino en el grupo de niños menores de 3 años, no hallándose diferencias en los de mayor edad.

El desarrollo de la DA es el resultado de la interrelación de factores genéticos, y ambientales producto de la exposición a múltiples alergenos.

El antecedente familiar de atopía constituye el componente genético más estudiado.

Existe consenso universal acerca de que la presencia de determinados genes predispone al desarrollo de DA como lo demuestra un estudio efectuado en mellizos ${ }^{21}$. En la actualidad se dispone de múltiples estudios que confirman la asociación entre DA y AF de atopía ${ }^{8,10-12}$. Uehara y cols, reportaron que el $59 \%$ y el $81 \%$ de los niños con DA poseen uno o los dos progenitores afectados por la misma enfermedad, respectivamente $^{22}$. Nosotros encontramos que el $46,9 \%$ de los niños atópicos presentaron AF de atopía en familiares de primer grado. $\mathrm{El}$ análisis estadístico evidenció que la DA se asoció de manera estadísticamente significativa con el AF de atopía en familiares de primer grado y en familiares de primero y segundo grado en los 2 grupos etarios estudiados, y sólo con el AF en familiares de $2^{\circ}$ grado en los niños menores de 3 años. Este hallazgo señalaría que se requeriría la presencia del antecedente de atopía en padres o hermanos para persistir con DA pasados los 36 meses de edad.

Clásicamente se acepta que la prevalencia de DA es menor en niños pertenecientes a familias numerosas. Este hallazgo sería consecuencia de una mayor exposición a infecciones a edades tempranas que actuarían modulando la respuesta inmune y disminuyendo así el riesgo de desarrollar $\mathrm{DA}^{23-25}$. Flohr y cols, en revisiones recientes sostienen que la relación entre el tamaño familiar y la DA sería consecuencia de la exposición a estímulos microbianos crónicos o recurrentes, no patogénicos y que el riesgo se incrementaría con el uso indiscriminado de antibióticos a edades tempranas, en especial en niños con AF de atopía ${ }^{26,27}$. El tamaño familiar no se asoció con la presencia de DA en los niños que evaluamos, a diferencia de lo encontrado por Sebok y cols, en un estudio llevado a cabo en Hungría, donde la DA fue más prevalente en las familias con $\leq 4$ integrantes ${ }^{12}$.

La vestimenta es un factor provocador del desarrollo de DA. Se conoce que el contacto directo de la lana con la piel exacerba la DA. El efecto irritativo de las fibras constituye, aún hoy, un motivo de discusión. Diepgen y cols, evaluaron el poder irritativo de diferentes materiales textiles y encontraron que las prendas de fibras sintéticas poseen un efecto irritativo significativamente mayor en pacientes con DA en comparación con las prendas de algodón, que fueron mejor toleradas ${ }^{28}$. La irritación también resultó dependiente del diámetro de la fibra sintética evaluada.

En nuestro estudio el contacto directo entre las vestimentas confeccionadas con fibras sintéticas y la piel se relacionó con la DA de forma estadísticamente significativa en los 2 grupos evaluados.

La relación existente entre la DA y el consumo de tabaco por parte de los convivientes de los pacientes es discutida. Existen trabajos 
que avalan el efecto desencadenante de la DA por parte de la inhalación pasiva del humo del tabaco $^{29,30}$. Si bien en el análisis univariado el consumo de tabaco dentro del hogar se asoció con la DA en los niños más pequeños de nuestra investigación, el tabaquismo no resultó un factor de riesgo al incluírlo en el análisis de regresión logística, coincidiendo con Dotterud y $\operatorname{col}^{9}$ y Sebok y $\operatorname{col}^{12}$, autores que tampoco encontraron esta asociación.

Ciertos alergenos inhalados como los ácaros presentes dentro del hogar han sido implicados en la etiopatogenia de la $\mathrm{DA}^{1}$.

Se han encontrado concentraciones de Dermatophagoides pteryonissimus diez veces superiores en hogares de pacientes atópicos al compararlos con viviendas de los sujetos controll $^{31}$.

Las alfombras constituyen un sitio donde se encuentra una elevada concentración de Dermatophagoides. Existen reportados estudios que evalúan medidas tendientes a disminuir la carga ambiental de este agente mediante la utilización de cobertores de colchones, aspiradoras de alto filtrado y aerosoles con efecto acaricida que demostraron la disminución de la severidad de la DA en el grupo en que se implementaron estas medidas ${ }^{32,33}$.

Como citan Tan y cols, la contribución de los ácaros en la etiología de la DA es aceptada popularmente, discutida por los alergistas y vista con escepticismo dentro del ambiente dermatológico ${ }^{32}$.

En nuestra investigación la existencia de alfombras dentro del hogar se asoció de manera estadísticamente significativa con la presencia de DA sólo en los niños menores de 3 años, probablemente debido a que permanecen mayormente dentro de la vivienda y a que aún no alcanzaron el desarrollo completo de su aparato respiratorio.

La contaminación ambiental producto del aumento de la industrialización y de la urbanización constituye un factor determinante del incremento de la prevalencia de DA en los últimos años ${ }^{1,2}$.

El ozono, el benceno, el dióxido de sulfuro, los metales pesados (níquel, cobre, arsénico y aluminio, entre otros) y sus subproductos que constituyen parte de los compuestos contami- nantes del aire son conocidos como irritantes de las vías respiratorias y potentes agentes proinflamatorios ${ }^{9,34}$. Un estudio reciente publicado por Morgenstern y cols ${ }^{35}$, constataron la presencia de asociación entre DA y habitar en las cercanías a una avenida, siendo dicha asociación de mayor significación si la vivienda se sitúa a menos de 50 metros de la calle en cuestión.

De los factores contaminantes evaluados en nuestra muestra, solamente habitar en las proximidades de una fábrica se asoció con la presencia de DA en ambos grupos etarios. Cabe destacar, sin embargo, que habitar en las cercanías de una fábrica aumentaría el riesgo de persistencia de la enfermedad en los niños de mayor edad, lo cual podría atribuírse a que desarrollan sus actividades recreativas principalmete fuera del hogar, teniendo mayor exposición a diferentes agentes contaminantes.

Contrariamente Dotterud y cols, encontraron que la prevalencia de DA en niños residentes en un área del Ártico ruso con altos niveles de contaminación del aire fue menor en comparación a otros estudios del norte de Europa ${ }^{9}$.

El escaso número de publicaciones sobre DA en la Argentina, la carencia de estudios epidemiológicos nacionales actualizados referidos al tema, como así también lo acotado del tamaño muestral del presente trabajo resultan hechos más que trascendentes para continuar investigando acerca de esta entidad dermatológica frecuente, y cada vez más prevalente entre los niños para, de acuerdo a resultados, poder sacar conclusiones.

La realización de un número importante de investigaciones serias sobre esta patología en nuestro medio nos permitirá conocer y poder analizar el comportamiento de la misma en los pacientes que atendemos, como así también poder comparar nuestra realidad con la de aquellos países que cuentan con información confiable sobre esta enfermedad.

\section{Conclusión}

Se encontró una alta prevalencia de DA, en especial en los niños mayores de 3 años. La DA afectó mayormente a los varones en el 
grupo de niños pequeños, no habiéndose constatado diferencias entre sexos entre los niños mayores.

De los factores de riesgo evaluados el $\mathrm{AF}$ de atopía, el uso de ropa de material sintético en contacto directo con la piel y habitar en las cercanías de una fábrica se relacionaron con la DA en ambos grupos etarios, en tanto poseer alfombra/s dentro del hogar se asoció con la enfermedad sólo en los niños pequeños.

Dada la escasez de estudios epidemiológicos de DA en nuestro medio creemos conveniente fomentar la realización de nuevas investigaciones para corroborar nuestros hallazgos.

\section{Referencias}

1.- Levy RM, Gelfand JM, Yan AC: The epidemiology of atopic dermatitis. Clin Dermatol 2003; 21 (2): 109-15.

2.- Santos Muñoz A: Dermatitis atópica. Preaderm. Soc Argent Dermatol 2003; Mód 1: 1-48.

3.- Pueyo ST, García MA, García Díaz R, Castro C, et al: Consenso Nacional de Dermatitis Atópica. Soc Argent Dermatol 2004: 1-37.

4.- Williams HC, Burney PG, Strachan D, Hay RJ: The U.K. Working Party's Diagnostic Criteria for Atopic Dermatitis. II. Observer variation of clinical diagnosis and signs of atopic dermatitis. Br J Dermatol 1994; 131 (3): 397-405.

5.- Dei-Cas I, Dei-Cas P, Acuña K: Atopic dermatitis and risk factors in poor children from Great Buenos Aires, Argentina. Clin Exp Dermatol 2009; 34 (3): 299-303.

6.- Williams $H$, Robertson $C$, Stewart A, et al: Worldwide variations in the prevalence of symptoms of atopic eczema in the International Study of Asthma and Allergies in Childhood. J Allergy Clin Immunol 1999; 103 (1 Pt 1): 125-38.

7.- Illi S, von Mutius E, Lau S, et al; Multicenter Allergy Study Group: The natural course of atopic dermatitis from birth to age 7 years and the association with asthma. J Allergy Clin Immunol 2004; 113 (5): 925-31.

8.- Johnke H, Vach W, Norberg LA, Bindslev-Jensen C, Host A, Andersen KE: A comparison between criteria for diagnosing atopic eczema in infants. Br J Dermatol 2005; 153 (2): 352-8.

9.- Dotterud LK, Falk ES: Atopic disease among adults in Northern Russia, an area with heavy air pollution. Acta Derm Venereol 1999; 79 (6): 448-50.

10.- Moore MM, Rifas-Shiman SL, Rich-Edwards JW, et al:
Perinatal predictors of atopic dermatitis occurring in the first six months of life. Pediatrics 2004; 113 (3 Pt 1): 468-74.

11.- Ricci G, Patrizi A, Baldi E, Menna G, Tabanelli M, Masi $M$ : Long-term follow-up of atopic dermatitis: retrospective analysis of related risk factors and association with concomitant allergic diseases. J Am Acad Dermatol 2006; 55 (5): 765-71.

12.- Sebok B, Schneider I, Harangi F; Primary Care Paediatricians in Baranya County: Familiar and environmental factors influencing atopic dermatitis in the childhood. J Eur Acad Dermatol Venereol 2006; 20 (4): 418-22.

13.- Halkjaer LB, Loland L, Buchvald FF, et al: Development of atopic dermatitis during the first 3 years of life: the Copenhagen prospective study on asthma in childhood cohort study in high-risk children. Arch Dermatol 2006; 142 (5): 561-6.

14.- Williams HC: Epidemiology of atopic dermatitis. Clin Exp Dermatol 2000; 25 (7): 522-9.

15.- Williams HC, Pembroke AC, Forsdyke H, Boodoo G, Hay RJ, Burney PG: London-born black Caribbean children are at increased risk of atopic dermatitis. J Am Acad Dermatol 1995; 32 (2 Pt 1): 212-7.

16.- George S, Berth-Jones J, Graham-Brown RA: A possible explanation for the increased referral of atopic dermatitis from the Asian community in Leicester. Br J Dermatol 1997; 136 (4): 494-7.

17.- Neame RL, Berth-Jones J, Kurinczuk JJ, GrahamBrown RA: Prevalence of atopic dermatitis in Leicester: a study of methodology and examination of possible ethnic variation. Br J Dermatol 1995; 132 (5): 772-7.

18.- Bohme M, Svensson A, Kull I, Nordvall SL, Wahlgren $C F$ : Clinical features of atopic dermatitis at two years of age: a prospective, population-based case-control study. Acta Derm Venereol 2001; 81 (3): 193-7.

19.- Wadonda-Kabondo N, Sterne JA, Golding J, Kennedy CT, Archer CB, Dunnill MG; ALSPAC Study Team: A prospective study of the prevalence and incidence of atopic dermatitis in children aged 0-42 months. Br J Dermatol 2003; 149 (5): 1023-8.

20.- Harris JM, Cullinan P, Williams HC, et al: Environmental associations with eczema in early life. Br J Dermatol 2001; 144 (4): 795-802.

21.- Schultz Larsen F, Holm NV, Hennigsen K: Atopic dermatitis: a genetic-epidemiologic study in a populationbased twin sample. J Am Acad Dermatol 1986; 15: 487-94.

22.- Uehara M, Kimura $C$ : Descendant family history of atopic dermatitis. Acta Derm Venereol (Stockh) 1993; 73: 62-3. 
23.- Matricardi PM, Rosmini F, Ferrigno L, et al: Cross sectional retrospective study of prevalence of atopy among Italian military students with antibodies against hepatitis A virus. BMJ 1997; 314 (7086): 999-1003.

24.- Linneberg A, Ostergaard C, Tvede $M$, et al: $\operatorname{IgG}$ antibodies against microorganisms and atopic disease in Danish adults: the Copenhagen Allergy Study. J Allergy Clin Immunol 2003; 111 (4): 847-53.

25.- Strachan DP: Hay fever, hygiene, and household size. BMJ 1989; 299 (6710): 1259-60.

26.- Flohr C, Pascoe D, Williams HC: Atopic dermatitis and the 'hygiene hypothesis': too clean to be true? Br J Dermatol 2005; 152 (2): 202-16.

27.- Williams H, Flohr C: How epidemiology has challenged 3 prevailing concepts about atopic dermatitis. J Allergy Clin Immunol 2006; 118 (1): 209-13.

28.- Diepgen TL, Stabler A, Hornstein OP: Textile intolerance in atopic eczema-a controlled clinical study. Z Hautkr 1990; 65 (10): 907-10.

29.- Brabäck L, Kälvesten L: Urban living as a risk factor for atopic sensitization in Swedish schoolchildren. Pediatr
Alergy Immunol 1991; 2: 14-9.

30.- Dotterud LK, Odland JO, Falk ES: Atopic dermatitis and respiratory symptoms in Russian and northern Norwegian school children: a comparison study in two arctic areas and the impact of environmental factors. J Eur Acad Dermatol Venereol 2004; 18 (2): 131-6.

31.- Beck HI, Korsgaard J: Atopic dermatitis and house dust mites. Br J Dermatol 1989; 120: 241-5.

32.- Tan BB, Weald D, Strickland I, Friedmann PS: Doubleblind controlled trial of effect of housedust-mite allergen avoidance on atopic dermatitis. Lancet 1996; 347 (8993): 15-8.

33.- Ricci G, Patrizi A, Specchia F, et al: Effect of house dust mite avoidance measures in children with atopic dermatitis. Br J Dermatol 2000; 143 (2): 379-84.

34.- Sublett JL: The environment and risk factors for atopy. Curr Allergy Asthma Rep 2005; 5 (6): 445-50.

35.- Morgenstern V, Zutavern A, Cyrys J, Brochow I, Koletzko S: Atopic diseases, allergic sensitization, and exposure to traffic-related air pollution in children. Am J Respir Crit Care Med 2008; 177: 1331-7. 